\title{
DECOUPLING CONTROL FOR THREE DEGREES OF FREEDOM SERVO SYSTEM BASED ON NEURAL NETWORK
}

\author{
${ }^{1}$ XIAOFEN JIA, ${ }^{2}$ BAITING ZHAO \\ ${ }^{1,2}$ Electrical and Information Engineering College, Anhui University of Science and Technology, Huainan, \\ China, 232001 \\ E-mail: ${ }^{1}$ jxfzbt2008@163.com, ${ }^{2}$ zhaobaiting@126.com
}

\begin{abstract}
Three degrees of freedom servo system(TDFSS) is one of the key equipments of inertial testing, such as evaluation of inertial navigation system and test of inertial components. It is a kind of servo system with some non-linearity and uncertainty. This thesis takes advantage of the characteristic of Neural network in approaching non-linear function, applies the Neural network on the three-axis simulator, provides a method for the TDFSS. Simulating experiment has been used to verify the advantage of the scheme and achieved completely decoupling control. The scheme gives good control precision, and it is simply structured and easily implemented.
\end{abstract}

Keywords: Three Degrees Of Freedom(TDF); Servo System(SS); Neural Network(NN); Decoupling Control(DC)

\section{INTRODUCTION}

TDFSS is one of the key equipment with the performances of high precision, fast response and low speed, which used for inertial testing and flying simulation in aviation aerospace field. In the operation of the simulator process, with the rise of axis swing frequency and swinging rate the mutual coupling disturbance torque is more and more bigger which will seriously affect the location accuracy and table rate stability of rotary. In conventional PID control-strategy, TDFSS of the axis can achieve good control characteristic and the system can achieve the desired design indexes, but when the servo system running in three degrees of freedom, with the improved speed of inner linkage of axial and central axis of rotation, the coupling between axis is enhanced, the simple PID controlstrategy is difficult to guarantee good control performance. It needs to consider decoupling control for the servo system based on the above reasons. Aiming at TDFSS decoupling control is a decoupling control problem of cultivatable variables system.

Cultivatable variables system of decoupling control has been a hot spot in controlled field for its wide application range, including aerospace, deep space explorations, power system, induction motor control, robots, chemical and pharmaceutical industries. At present there are many theory researches of this aspect, mainly including feedback liberalization methods based on differential geometry theories or inverse system thoughts. However, these methods usually demand that the mathematic model of the system is clearly known while in actual project the controlled object of nonlinear is difficult to be described exactly, and the controlled object is often gray box even blackbox system. Therefore the methods above are hard to be applied in practice.

In recent years, artificial neural network is used in identification and controlling of cultivatable systems and has made great progress. This paper will research TDFSS decoupling control system based on artificial neural network.

\section{RBF NETWORK STRUCTURE}

Radial basis function neural network (RBF) is a three layer with a single hidden layer feed-forward network. It has been proven RBF neural network can approach a given nonlinear function. RBF neural network has global optimization and best approximation of the performance.

The output of RBF neural network is as follows,

$$
y=f_{i}(x)=\sum_{K=1}^{N} W_{i k} \phi_{K}\left(\left\|x-C_{K}\right\|^{2}\right)
$$


Gaussian function is used as radial basis function as follows,

$$
\phi_{K}(x)=\exp \left(-\frac{\left\|x-C_{K}\right\|^{2}}{2 \sigma_{K}^{2}}\right)
$$

In Gaussian core function, $X$ is input and independent variable vector, $C$ is the center of RBF and constant vector. $\Phi(x-c)$ is radial basis function. RBF-based network model is shown in Fig.1.

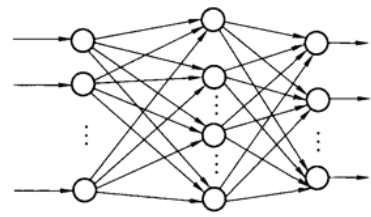

Input Layer Hidden Layer Output Layer

Fig.1. RBF-based network model

Network parameter update according to the following formula:

$$
\begin{gathered}
W(n+1)=W(n)+\mu_{W} e(n) \varphi(n) \\
C_{K}(n+1)=C_{K}(n)+\mu_{C} \frac{e(n) W_{K}(n)}{\sigma_{K}{ }^{2}(n)} \\
\varphi\left[x(n), C_{K}(n), \sigma_{K}\right]\left[x(n)-C_{K}(n)\right] \\
\sigma_{K}(n+1)=\sigma_{K}(n)+\mu_{\sigma} \frac{e(n) W_{K}(n)}{\sigma_{K}{ }^{2}(n)} \\
\varphi\left[x(n), C_{K}(n), \sigma_{K}\right]\left\|\left[x(n)-C_{K}(n)\right]\right\|^{2}
\end{gathered}
$$

\section{THE CONTROL STRUCTURE OF TDFSS BASED ON RBF NETWORKT}

\subsection{Neural Network Decoupling Control}

Using conventional PID control strategy, when the three-axis rotary joint in three axis winding, as the axis swing frequency and swinging rate rising, the improvement of the coupling effect between each axis increased, the simple PID control strategy is difficult to ensure better control performance, therefore it need to design decoupling controller to compensate for the coupling between the axes. Figure 2 and Figure 3 shows the coupling effect to other axes when central and the inner axes system carry sinusoidal. Curve indicated by the figure of the coupling of the outer ring of the rotary axis positioning accuracy can not meet specifications.

The RBF neural network can approach the inverse dynamics of TDFSS perfectly, so it been used to design the decoupling control system based on the existing control system.

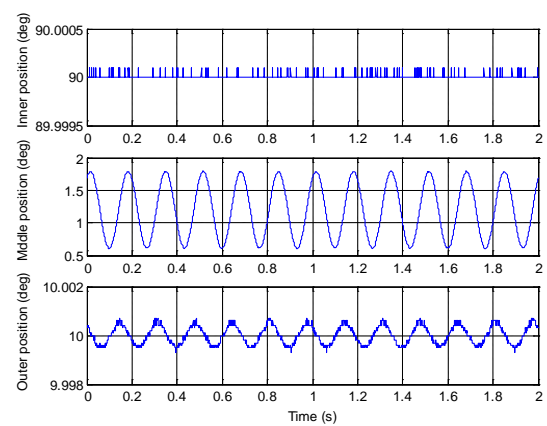

Fig.2. Central Axis Of The Coupling Of The Other Two Axes

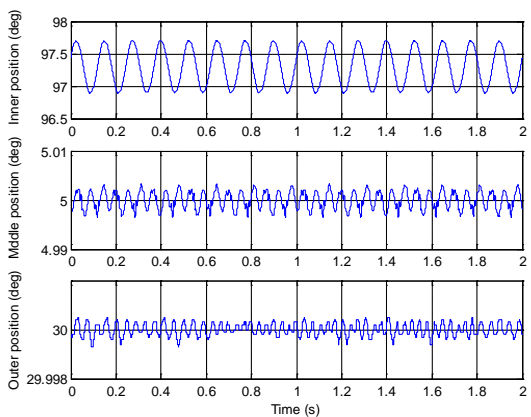

Fig.3. Inner Axis Of The Coupling Of The Other Two Axes

\section{2 Nverse Modeling Of Three-axis Simulator}

In order to achieve the decoupling control of TDFSS, first the inverse of servo system modeling. This will use RBF networks to achieve servo system for the inverse dynamic identification.

Neural network inverse dynamic modeling of the structure shown in Figure 4.RBF networks with one hidden layer approximation of inverse dynamic model of TDFSS, Three degree angular position signal $[\alpha \beta \gamma]_{\text {Angular velocity signal }}\left[\begin{array}{lll}\dot{\alpha} & \dot{\beta} & \dot{\gamma}\end{array}\right]_{\text {as }}$ the network input signal. Network output signal for the amount of control axes. $\left[\begin{array}{lll}u_{a_{i}} & u_{a_{m}} & u_{a_{o}}\end{array}\right]$.So the network has 9 input nodes and 3 output nodes. The number of the selecting network's hidden layer neuron is 40. At the same times, Selecting $n_{u}=3 n_{y}=3$

Hidden layer neuron activation function is a hyperbolic tangent function, and its expression is as follows ceremony.

$$
f(x)=\tanh (x)=1-\frac{1-e^{-x}}{1+e^{-x}}
$$




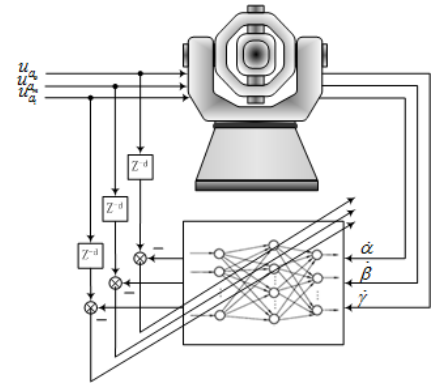

Fig.4. Neural Network Model Structure

\section{3 Network Learning Algorithm}

Radial basis function (RBF) network algorithm procedure is as follows:

1. Select a group initial center value $C_{K}$ from the input vector;

2. Variance value calculation

$$
\sigma=\frac{d_{\max }}{K}
$$

$d_{\max }$ is the greatest distance, $K$ is the number of $C_{K}$.

3. Calculate the $\hat{y}_{i}(n)$ by $x(n)$

$$
\hat{y}_{i}(n)=\sum_{k=1}^{M} W_{K} \phi\left[x(n), C_{K}, \sigma_{K}\right]
$$

4. Update the network parameters according to the Formula (5-7).

5. If network convergence, stop the calculation, or turn to step 4.

In order to learn neural network inverse dynamic model of TDFSS better, the input signal used to excite the system should contain a rich frequency response, to ensure network convergence in a relatively short period of time. Usually take the input signal or pseudo-random signal sinusoidal signal. As the servo system bands were $8 \mathrm{HZ}, 6 \mathrm{HZ}$ and $4 \mathrm{HZ}$. So select the $0.1 \sim 15 \mathrm{HZ}$ sine signal as stimulus. Use 400 groups of data as training samples, the network's training goals selected 0.005 . After 3478 times iterations of the network convergence. Figure 5 shows the network training error curve. Figure 5 shows the network training error curve.

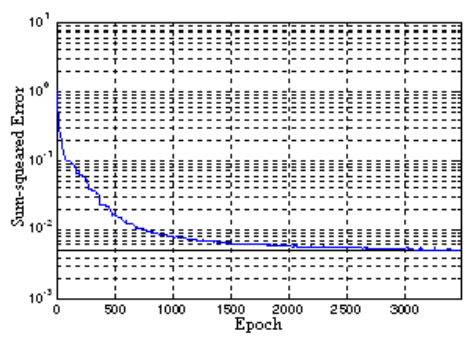

Fig.5. Training Error Curve

\subsection{Neural Network Decoupling Control}

RBF network decoupling scheme is used to achieve the decoupling control of TDFSS.

Algorithm is taking advantage RBF network training.

Simulation uses MatLab. Supposing the central ring and inner ring respectively the input signals $2 \sin (2 \pi \cdot 6 \cdot t)$ and $2.5 \sin (2 \pi \cdot 8 \cdot t)$, it requires outer ring tracking the signal 0.1 degree per second. The simulation results are shown in Figure 6. Figure 6a is a system in which control strategies in the outer PID rate curves, and Figure $6 \mathrm{~b}$ is the rate of outer ring outputs curves using neural network decoupling control strategy. Supposing the central ring and inner ring respectively the input signals $2 \sin (2 \pi \cdot 2 \cdot t)$ and $2.5 \sin (2 \pi \cdot 3 \cdot t)$, it requires outer ring being in the position servo state . The simulation results are shown in Figure 7. Figure 7a is a system in which control strategies in the outer PID rate curves, Figure $7 \mathrm{~b}$ is the use of neural network decoupling control strategy, the rate of the output curve of the outer ring.

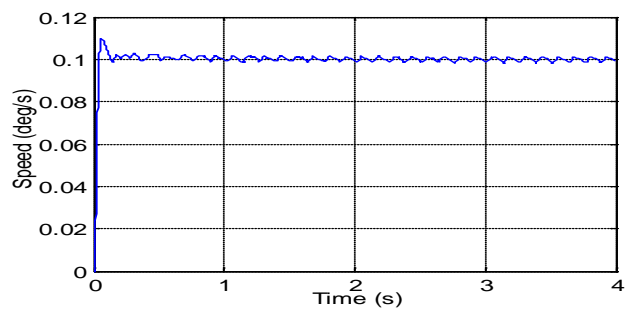

(a)

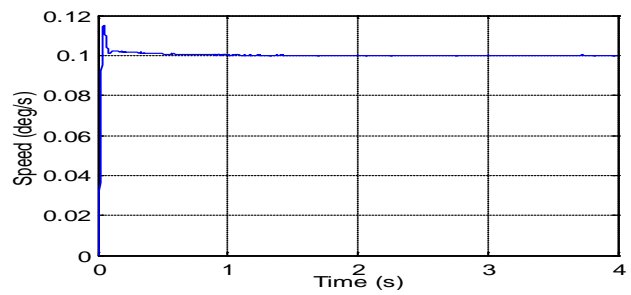

(b)

Fig.6. Outer Speed Curve In Different Control Strategies

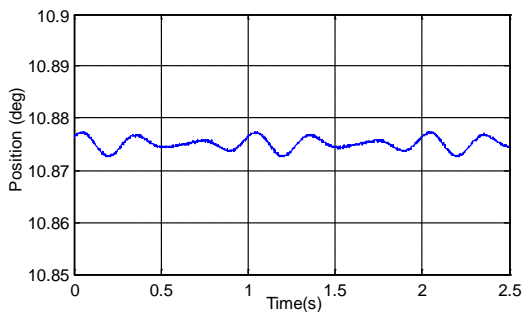

(A) 


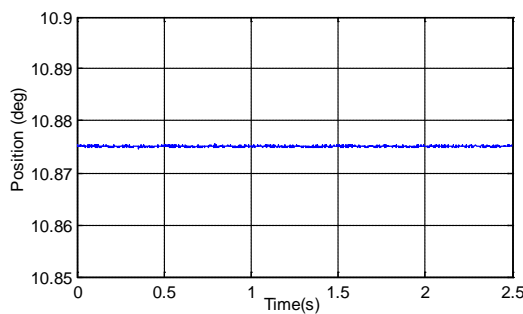

(B)

Figure 7 Outer Speed Curve In Different Control Strategies

The simulation results show that using neural network decoupling control, the output of outer ring state rate from the interference of others and the system achieve a complete decoupling.

\section{CONCLUSION}

This paper was introduced the RBF neural network into the multivariable nonlinear controlling, and accurately identify the inverse dynamic model of TDFSS using neural network. And it was used in strategy of decoupling control, thus was achieved the neural network decoupling control of TDFSS. Using the actual dynamic model of TDFSS and its simulation results can validate the feasibility of which use neural network control to the TDFSS decoupling.

\section{ACKNOWLEDGEMENT}

The research is supported by the Youth Foundati on of Anhui University of science \& technology of China under Grant No.12257, No.2012QNZ06, the Doctor Foundation of Anhui University of science \& technology of China under Grant No.11223, and the Guidance Science and Technology Plan Project s of Huainan under Grant No.2011B31

\section{REFERENCES}

[1] Chemachema, Mohamed. Belarbi, Khaled. "Direct adaptive neural network controller for a class of nonlinear systems based on fuzzy estimator of the control error", International Journal of Systems Science , Vol. 42, No. 7, July 2011, pp. 1165-1173.

[2] Ho, M.L. ; Chan, P.T.; Rad, A.B.; Shirazi, M.; Cina, M. " A novel fused neural network controller for lateral control of autonomous vehicles ", Applied Soft Computing Journal, Vol. 12, No. 11, November 2012, pp. 35143525.
[3] Mitchell, Ryan. Kim, Yeesock. El-Korchi, Tahar. "System identification of smart structures using a wavelet neuro-fuzzy model ", Smart Materials and Structures, v 21, n 11, November 2012

[4] May, P. Zhou, E. Lee, C.W. " Learning in fully recurrent neural networks by approaching tangent planes to constraint surfaces " , Neural Networks, Vol. 34, October 2012 ,pp. 72-79.

[5] Yi Guoxing. " A study on Design Problems of Three-Axis Simulator", Harbin Institute of Technology, 2005.

[6] Li Wei. Ren, Shun Qing. Zhao Hong-Bo. "Influence of three-axis turntable error on gyro calibration accuracy ", Electric Machines and Control, Vol.15, No.10, October 2011, pp.101106.

[7] Liu Qiaosheng. Xi Juntong. " Case-based parametric design system for test turntable ", Expert Systems with Applications, Vol.38, No.6, June 2011, pp.6508-6516. 